\title{
Changes in the GNSS precise point positioning accuracy during a strong geomagnetic storm
}

\author{
Anna Yasyukevich*, Semen Syrovatskii, and Yury Yasyukevich \\ Institute of Solar-Terrestrial Physics SB RAS, 664033 Irkutsk, Russia
}

\begin{abstract}
Based on the data from dual-frequency receivers of global navigation satellite systems (GNSS), we analyze the changes in GNSS positioning accuracy during the August 25-26, 2018 strong geomagnetic storm on a global scale. The storm is one of the strongest geomagnetic events of the solar cycle 24 . To analyze the positioning quality, we calculated coordinates using the precise point positioning (PPP) method in the kinematic mode. We recorder a significant degradation in the PPP positioning accuracy during the main phase of the storm. The maximum effect is observed in the middle and high latitudes of the US-Atlantic longitude sector. The average PPP error during the storm is shown to exceed $\sim 0.5 \mathrm{~m}$, that is up to 5 times higher than the values typical on quiet days. Areas with increased PPP errors is revealed to correspond to the regions with significant increase in the intensity of total electron content variations of 10 20 min period range. This increase is presumably due to the auroral oval expansion toward middle latitudes.
\end{abstract}

\section{Introduction}

Global navigation satellite systems (GNSS) have become tightly integrated into human daily lives and economic activity. The state of the ionosphere is one of crucial factors for the GNSS positioning accuracy. While the navigation signal propagates through the ionosphere, ionospheric irregularities can lead to the signals scattering [1]. The effect is more pronounced when the irregularity scale is of the radiowave first Fresnel zone $\sim \sqrt{\lambda \mathrm{Z}}$ (where $\lambda$ is the signal wavelength, $Z$ is the distance to the irregularity). It is about $100-300 \mathrm{~m}$ in GNSS case, i.e. socalled small-scale irregularities. The scattering leads to radio signal amplitude scintillations and, in the extreme case, may give rise to signal losses-of-lock [2]. Deep signal fades appearing due to small-scale irregularities result in navigation outages [3]. This can decrease the stability of the GNSS service and even arouse inability to provide coordinates to a user.

In quiet geophysical conditions, ionospheric irregularities of different scales can appear in high-latitude and equatorial regions. The amplitude scintillations and GNSS signal lossesof-lock are often observed in these regions [4]. Geomagnetic storms are known to be a source for small-scale ionospheric irregularities in wide latitudinal range. Geomagnetic storms, as well, feature occurrence of traveling ionospheric disturbances (TID) propagating from auroral oval toward lower latitudes. TIDs also can be a source for GNSS positioning quality

* Corresponding author: annpol@,iszf.irk.ru 
deterioration. Luo et al. [5] studied several strong storms during solar cycle 24, including the 2015 March 17 St. Patrick storm. They reported increase in precise point positioning (PPP) error during the storm up to $0.32 \mathrm{~m}$ at medium and above $1 \mathrm{~m}$ at high latitudes with respect to only $0.163 \mathrm{~m}$ on a quiet day.

In this study, we analyse the dynamics in GNSS positioning error during the August 2526,2020 strong geomagnetic storm on a global scale. Our task is to identify the changes in positioning accuracy in different latitudinal-longitudinal sectors depending on the storm evolution.

\section{PPP error estimating}

We study is based on the data of phase dual-frequency measurements from the worldwide GNSS network [6]. To estimate the changes in positioning accuracy, for every station we calculated GPS precise point positioning (PPP) coordinates in the kinematic mode. The calculating was performed using the GAMP opensource software [7]. Receiver and satellite clock offsets are considered in GAMP PPP solution by applying IGS precise satellite orbit and clock products. Besides, linearized equations of original pseudorange and carrier phase observations take into account line-of-sight (LOS) ionospheric delay, receiver non-calibrated code delay, receiver and satellite non-calibrated phase delays, and zenith wet delay.

We used a dual-frequency PPP model, in which the receiver non-calibrated code delays are absorbed by both receiver clock offset and LOS ionospheric delay parameters. The 24hour averaged values of $\mathrm{X}, \mathrm{Y}, \mathrm{Z}$ coordinates for a station were regarded as reference positions. The total positioning error was calculated as a difference between the reference and the instant position:

$$
\sigma_{X Y Z}=\sqrt{\left(\Delta X^{2}+\Delta Y^{2}+\Delta Z^{2}\right)}
$$

To estimate the dynamics of the error in different latitudinal regions, we calculated zonal mean error values for the European-Asian $\left(0-180^{\circ} \mathrm{E}\right)$ and American-Atlantic (- 180:0 $\left.\mathrm{E}\right)$ sectors within the latitude bins of $2.5^{\circ}$ size.

\section{Geomagnetic storm of August 25-26, 2018}

We consider the PPP error dynamics during the August 25-26, 2018 geomagnetic storm. The storm is one of the strongest geomagnetic events of the solar cycle 24. The storm was triggered by the August 20, 2018 Coronal Mass Ejection (CME). On August 25, 2018 the CME reached the Earth, giving rise to a strong G3 geomagnetic storm. Authors [8] studied CME propagation in the interplanetary space and analysed its effects in the magnetosphere and the ionosphere.

Right panel on Fig. 1 shows the dynamics in Sym-H index (in black) and Bz component (in red) of interplanetary magnetic field (IMF) during the storm. It can be seen that the IMF direction changed to southward at about 17:00 UT on August 25. At the same time, a sharp drop in the Sym-H index was observed, indicating storm-related evolution of magnetospheric ring current. The minimum Sym-H index value was recorded at 07:15 UT on August 26 and reached $-201 \mathrm{nTl}$, whereupon the storm recovery phase began.

Left panel on Fig. 1 demonstrates variations in the real-time indexes of auroral activity AE, AU, AL by the data from http://wdc.kugi.kyoto-u.ac.jp. A significant increase in auroral activity is seen during the storm, reaching a peak (AE $>2000 \mathrm{nTl})$ at about 07:40 UT on August 26, 2020. After that, there were several pulsations in auroral activity with clear peaks during the day. 

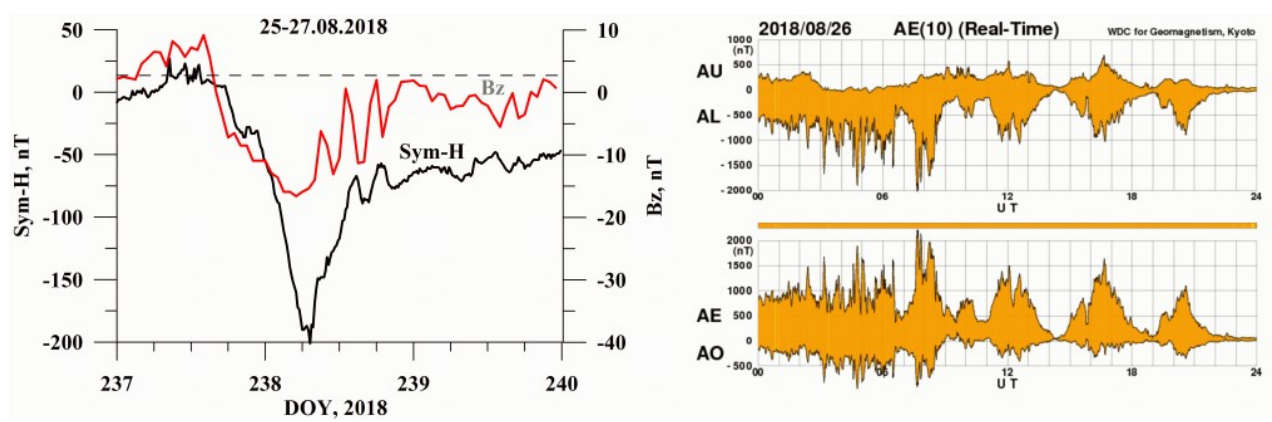

Fig. 1. Changes in: right - IMF Bz component (red line) and Sym-H (black line) index on August 2527, 2020; left - auroral activity indexes AE, AL, AU on August 26, 2020 (credit by http://wdc.kugi.kyoto-u.ac.jp)

\section{PPP errors during the storm}

Fig. 2 provides the distributions in PPP error around the globe at 07:45 UT on August 25 (c, before the storm) and on August 26 (d, during the storm main phase). Panels a and $b$ in the figure show the intensity in total electron content (TEC) variations filtered within 10-20 min range at the corresponding time instants. These variations are obtained using the SIMuRG tool for collecting, processing, storage and presentation of GNSS TEC data [9].

In the Fig. $2 b$, during the storm main phase, we can see a significant enhancement in TEC variation intensity in the areas stretched along the auroral oval boundaries, both in the Northern and Southern hemispheres. In the North American sector, strong disturbances reach $40^{\circ} \mathrm{N}$ latitude. Increased variations are also observed in the equatorial region. In these same areas, we can observe heightened values of total positioning error $\sigma_{X Y Z}$ (Fig. 2d) that were not recorded in the period before the storm. The error values exceed $0.5 \mathrm{~m}$ compared to $\sim 0.1 \mathrm{~m}$ on a quiet day.
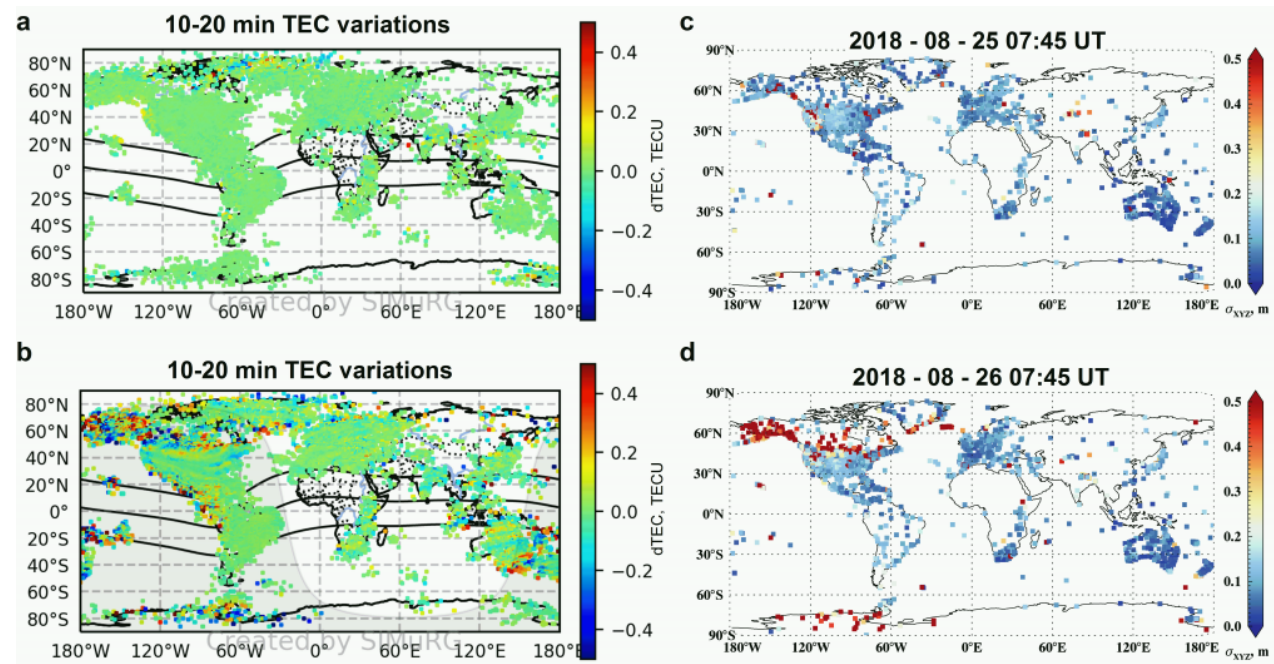

Fig. 2. Distributions in PPP error at 07:45 UT on August 25 (c) and August 26 (d). The intensity in TEC variations within 10-20 min period range at the corresponding time instances $(a, b)$. 
Fig. 3 shows the zonal mean values of total PPP error $\sigma_{X Y Z}$ averaged in American Atlantic (a-c) and Asian - European (d-f) longitudinal sectors. Panels a and d show the dynamics in PPP error for the reference quiet day (August, 24; 236 DOY), the panels b and e show the data for the storm beginning day (August 25, 237 DOY) and the panels $\mathrm{c}$ and $\mathrm{f}-$ for August 26 (238 DOY). Note that the quality of the GAMP PPP solution over 00-02 UT is unsatisfactory and we do not consider this time. The horizontal navy-blue strips on the panels (zero values) mark absence of the data.
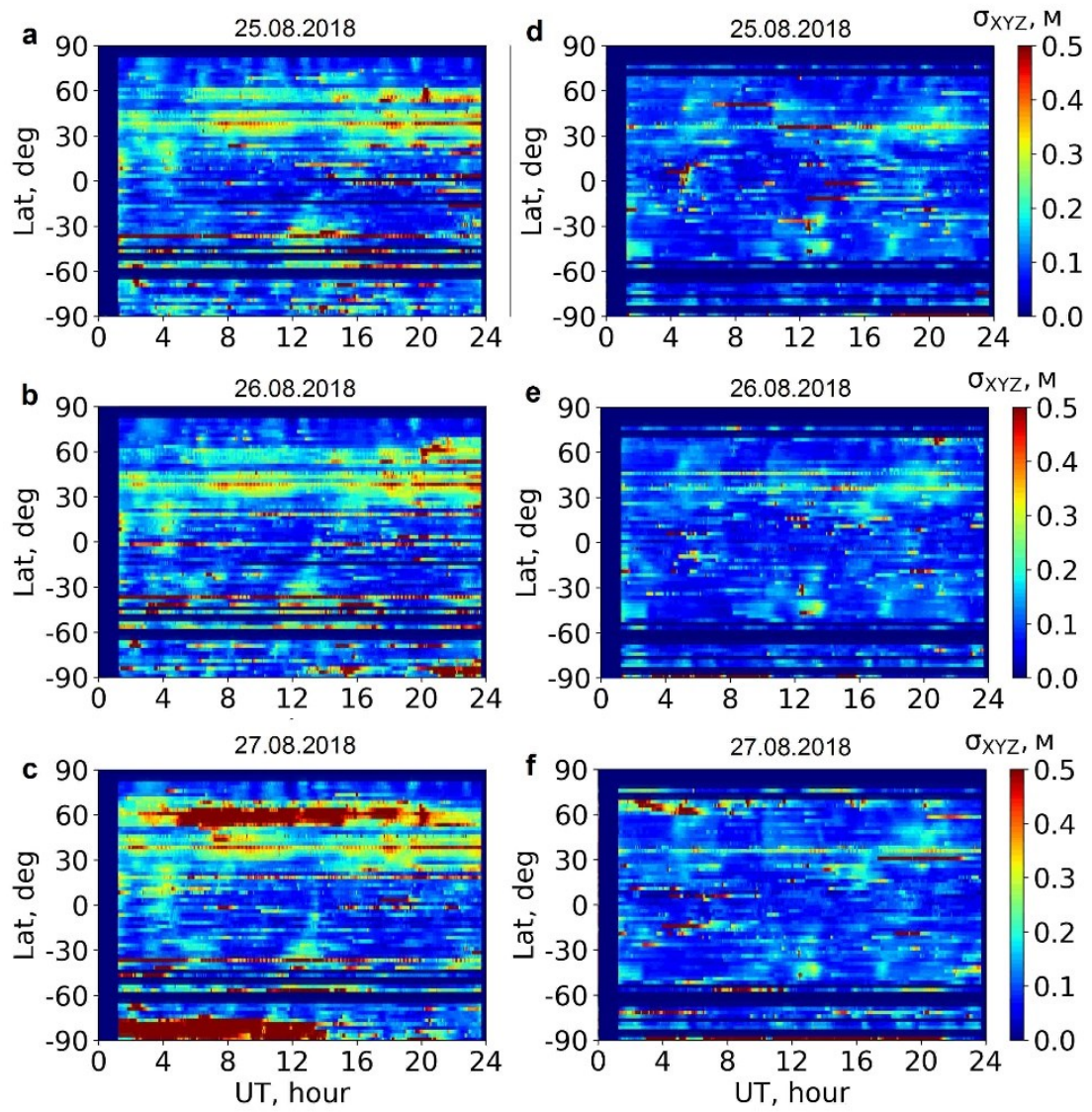

Fig. 3. Averaged total PPP error $\sigma_{X Y Z}$ vs latitude in the American - Atlantic (left) and Asian European (right) longitudinal sectors on August 25-27, 2020. The latitude bin size is $2.5^{\circ}$.

The results for the reference day show that the characteristic error in most regions do not exceed $0.1 \mathrm{~m}$. Increased error values are observed over $30-60^{\circ} \mathrm{N}$ latitudes in the American Atlantic sector. The error reaches $0.3 \mathrm{~m}$ here even on a quiet day. These PPP error increases are due to satellite geometry or data processing peculiarities. Such effects have similar patterns for both the storm and the reference days.

After 20:00 UT on August 25 we can notice a slight increase in the average error at 65$75^{\circ} \mathrm{N}$ latitudes in the Northern hemisphere both in American-Atlantic and Asia-European sectors. A sharp decrease in the PPP quality is clearly observed during all day on August 26 in the North American sector. The average PPP error is significantly greater than $0.5 \mathrm{~m}$. Also, a noticeable growth in PPP error is visible in high latitudes of the Southern hemisphere until 
14 UT. In the European-Asian sector, positioning deterioration is observed at $60-70^{\circ} \mathrm{N}$ latitudes till around noon.

So, we recorded a precision deterioration, which results in fivefold worse positioning accuracy during the storm. The revealed PPP positioning deterioration is comparable in magnitude with that observed during the September 6, 2018 solar radio flare [10] but lasts much longer. Our analysis has shown that this positioning quality degradation was observed even longer than during the St. Patrick magnetic storm. Authors [11] revealed that smallscale irregularities triggering GNSS positioning deterioration are most likely caused by an the auroral oval expansion during a storm.

The research is supported by the Russian Federation President Grant No. MK-3265.2019.5 as well as budgetary funding of Basic Research program II.16. TEC variation maps were obtained via SIMuRG on-line service (https://simurg.iszf.irk.ru). The SYM-H index data were provided by the WDC for Geomagnetism, Kyoto (http://wdc.kugi.kyoto-u.ac.jp/wdc/). We acknowledge use of NASA/GSFC's Space Physics Data Facility's OMNIWeb service, and OMNI data (https://omniweb.gsfc.nasa.gov).

\section{References}

1. K.C. Yeh, C.H. Liu, Proceedings IEEE, 70, 4, 324-360 (1982)

2. P. M. Kintner, B. M. Ledvina, E. R. de Paula, Space Weather, 5, S09003 (2007)

3. A.O. Akala, P.H. Doherty, C.S. Carrano, C.E. Valladares, K.M. Groves, Radio Sci., 47, RS4007 (2012)

4. Y. Jiao, Y.T. Morton, Radio Sci., 50, 886-903 (2015)

5. X. Luo, S. Gu, Y. Lou, C. Xiong, B. Chen, X. Jin, Sensors, 18, 1784 (2018)

6. J.M. Dow, R.E. Neilan, C. Rizos, J. Geodesy, 83, 191-198 (2009)

7. F. Zhou, D. Dong, W. Li, X. Jiang, J. Wickert, H. Schuh, GPS Solutions, 22, 33 (2018)

8. M. Piersanti, P. De Michelis, D. Del Moro, et al., Annales Geophysicae (to be published)

9. Y.V. Yasyukevich, A.V. Kiselev, I.V. Zhivetiev, I.K. Edemskiy, S.V. Syrovatskii, B.M. Maletckii, A.M. Vesnin, GPS Solutions, 24, 69 (2020)

10. Y. Yasyukevich, E. Astafyeva, A. Padokhin, V. Ivanova, S. Syrovatskii, A. Podlesnyi, Space Weather, 16, 1013-1027 (2018)

11. Y. Yasyukevich, R. Vasilyev, K. Ratovsky, A. Setov, M. Globa, S. Syrovatskii, A. Yasyukevich, A. Kiselev, A. Vesnin, Remote Sens., 12, 1579 (2020) 